

Carter, A., and Pritchard, D. (2016) Intellectual humility, knowledge-how, and disagreement. In: Mi, C., Slote, M. and Sosa, E. (eds.) Moral and Intellectual Virtues in Western and Chinese Philosophy: The Turn Toward Virtue. Routledge: New York, pp. 49-63. ISBN 9781138925168.

There may be differences between this version and the published version. You are advised to consult the publisher's version if you wish to cite from it.

http://eprints.gla.ac.uk/136257/

Deposited on: 6 February 2017

Enlighten - Research publications by members of the University of Glasgow http://eprints.gla.ac.uk 
For The Virtue Turn, (eds.) M. Slote, E. Sosa \& C. Mi, (Publisher TBC).

\title{
INTELLECTUAL HUMILITY, KNOWLEDGE-HOW, AND DISAGREEMENT
}

\author{
J. Adam Carter \& Duncan Pritchard \\ University of Edinburgh
}

\begin{abstract}
A familiar point in the literature on the epistemology of disagreement is that in the face of disagreement with a recognised epistemic peer the epistemically virtuous agent should adopt a stance of intellectual bumility. That is, the virtuous agent should take a conciliatory stance and reduce her commitment to the proposition under dispute (e.g., Elga 2007; Feldman 2004; Christensen 2007). In this paper, we ask the question of how such intellectual humility would manifest itself in a corresponding peer disagreement regarding knowledge-how. We argue that while it is relatively straightforward to recast this debate in terms of a reductive intellectualist account of knowledge-how (e.g., Stanley \& Williamson 2001; Stanley 2011a; 2011b; Brogaard 2008; 2009; 2011), whereby knowledge-how just is a matter of having a particular propositional attitude, the issue becomes more complex once we turn to anti-intellectualist positions (e.g., Ryle 1945; 1949; Poston 2009; Carter \& Pritchard 2013; 2014). On these views, after all, such a disagreement won't be just a matter of disagreeing about the truth of a proposition. Accordingly, to the extent that some kind of conciliation is plausibly required of the virtuous agent in the face of a recognised peer disagreement, this conciliation will not consist simply in belief revision. We propose a novel way to address this problem. We claim that what is required of the epistemically virtuous agent when confronted with peer disagreement regarding knowing how to $\varphi$ is that thereafter she should be disposed to employ her way of $\varphi$-ing across a narrower range of practical circumstances than beforehand. Moreover, just as an agent needs to call on her intellectual virtues in order to determine the extent of conciliation required in an ordinary case of epistemic peer disagreement, so the intellectual virtues will play an important role in determining this shift in dispositions to $\varphi$ that occurs as regards epistemic peer disagreement about knowledge-how.
\end{abstract}

\section{DISAGREEMENT AND INTELLECTUAL HUMILITY}

Disagreements are pervasive. In some cases, they are significant from the perspective of practical action-e.g., disagreement can constitute practical obstacles to accomplishing our objectives. ${ }^{1}$

But disagreements can also be significant from the perspective of what we should believe. In particular, it is widely held by epistemologists that if you believe that some proposition $p$ is true, then when someone else maintains that $p$ is false, this can (in some circumstances) be relevant for 
the purposes of whether you should continue believing $p$ to the same extent (where the 'should' here is broadly the should of epistemic rationality). ${ }^{2}$

The general statement that disagreements can matter, epistemically, needs some refining. This is because no one thinks that just any disagreement should be epistemically significant. To make this point concrete, just suppose that leading theoretical physicist Stephen Hawking believes a certain proposition about black holes—call this proposition ' $\mathrm{H}$ '. Now suppose Hawking were to find out that an untrained, first-year pupil of his purports to disagree with him about $\mathrm{H}$. Is this discovered disagreement rationally significant for Hawking in the sense that, from an epistemic point of view, Hawking should now be less confident now than he was before, that $\mathrm{H}$ is correct?

It's hard to see how. There are a number of things that we might be inclined to say here, but a very natural observation is that Hawking and the first-year student do not seem, prior to their disagreement, equally poised to be right. More specifically, it seems obvious that Hawking differs substantially from his interlocutor in that: (i) he has more evidence about whether $\mathrm{H}$ is true; and also, (ii) he has considerably greater cognitive ability in the arena of theorizing about matters such as $\mathrm{H}$ than does his student. It seems precisely because of these asymmetries that Hawking would be well within his rights to regard the pupil not as an epistemic peer with regard to the subject matter in question, but rather as his epistemic inferior, at least in this regard. ${ }^{3}$ Furthermore, in light of justifiably regarding his pupil as an epistemic inferior with regard to $\mathrm{H}$, it seems that Hawking is going to be epistemically blameless in simply carrying on believing $\mathrm{H}$ to the very same extent as before, even though his first-year pupil (perhaps even adamantly) disagrees with him. ${ }^{4}$

Suppose however, that we change the details of the scenario a bit. Let's keep everything else the same, but swap the first-year student for someone with (considerably) greater cognitive ability and evidence about black holes. Suppose, for example, that Hawking learns that renowned theoretical physicist (and frequent collaborator and co-author with Hawking) Roger Penrose disagrees with Hawking about whether $\mathrm{H}$ is true. It seems initially compelling that, in a situation like this, Hawking is not going to be equally blamelessness were he to simply_after learning that Penrose disagrees with him—carry on believing $\mathrm{H}$ to just same extent as before, when the disagreement was with his pupil. After all, Penrose, unlike the pupil, plausibly is an epistemic peer with Hawking in this respect, someone who (prior to the dispute) Hawking will have regardedon the basis of Penrose's evidence and abilities - to be (roughly) just as well poised to be right on black-hole related matters as Hawking himself. 
A widely shared insight in the disagreement literature is that, in the face of a disagreement with a recognised epistemic peer (such as between Hawking and Penrose), the epistemically virtuous agent should adopt a stance of intellectual bumility — that is, a stance where one exhibits some measure of epistemic deference by reducing one's initial confidence in the matter in contention. ${ }^{5}$ There is, to be sure, plenty of in-house dispute amongst those who share this broad insight just what this will involve. According to one particularly strong way to flesh out the insight, advanced by Adam Elga (2007), you should give equal weight to the view of your recognised epistemic peer as you do to your own view. In practice, this would mean that Hawking and Penrose should rather dramatically reduce their confidence in the target proposition, ultimately 'splitting the difference'. For Richard Feldman (2007), in contrast, the eventual result would be a withholding of judgement, by both parties. ${ }^{6}$

Even many opponents of stances as concessionary as Elga's and Feldman's, however, submit that under some circumstances discovering that a recognised epistemic peer disagrees with you is epistemically significant in the following sense: that you should no longer be just as confident with regard to the matter in question as before. ${ }^{7}$ As this line of thinking goes, the intellectually virtuous agent should thus exhibit some degree of epistemic humility in these circumstances. For the purposes of what follows, we shall leave open just how much conciliation this involves.

\section{INTELLECTUALISM, ANTI-INTELLECTUALISM AND KNOW-HOW DISAGREEMENTS}

Debates in social epistemology about peer disagreement uniformly concern propositional disagreements, where what is disagreed about is the truth of some target proposition. ${ }^{8}$ For example, in one much-discussed case offered by David Christensen (2007) involving 'mental math', the object of dispute is whether an individual's share of a restaurant bill is $\$ 43$. $^{9}$ One party thinks that it is, another thinks that it is not. Against the background where cases of like this are the point of focus, philosophical questions about the extent of intellectual humility that is called for in the face of recognised epistemic peer disagreement are naturally cast within the familiar language of belief revision-i.e., to what extent should one revise one's epistemic stance, with respect to the proposition is dispute?

But just as many disagreements we find in epistemic practice are disagreements about (say) whether something is so (and so, about whether some proposition $p$ is true), ${ }^{10}$ other equally 
genuine disagreements are about how to do things - e.g., how to $\phi$ - and some of these disagreements (just like any other disagreements) will feature recognised epistemic peers. ${ }^{11}$ For convenience sake, call these disagreements know-how disagreements: each party to the disagreement purports to know-how to $\phi$, and that the other party to the disagreement has got it wrong.

Just as there are superficial propositional disagreements, we can as easily imagine superficial know-how disagreements too. An example of the former could be when, in some context, Tim asserts that $\mathrm{x}$ is $\mathrm{F}$, and Tom asserts that $\mathrm{x}$ is $\mathrm{G}$, where $\mathrm{Fx}$ and $\mathrm{Gx}$ are not mutually exclusive (i.e., Tim and Tom are not asserting propositions with incompatible contents). An example of the latter could be when the disagreement is simply on the grounds that A and B are inclined to use distinct methods of $\phi$-ing-vi\%, where A proposes that $\phi$-ing can be accomplished by method $M$, and $\mathrm{B}$ proposes that $\phi$-ing can be accomplished by method N. It might be, after all, that $\mathrm{M}$ and $\mathrm{N}$ are regarded by both $\mathrm{A}$ and $\mathrm{B}$ as equally valid ways of $\phi$-ing. Thus mere proposals of different ways of $\phi$-ing needn't by itself constitute what we are calling a (genuine) know-how disagreement. What's needed is that one party regards the other party's proposed way of $\phi$-ing as incorrect. While we will be less inclined to locate know-how disagreements in cases where it is common ground to both parties of the dispute that there are multiple equally valid ways of $\phi$-ing, more fertile ground for such disagreements will be cases where it's common ground that there is some unique correct way to $\phi{ }^{12,13}$ We'll revisit this point below.

The kind of know-how disagreements that concern us will be ones involving epistemic peers. In line with our treatment of epistemic peers above, we will treat an epistemic peer in this case as being someone who is roughly epistemically alike both in terms of the possession of relevant evidence (i.e., concerning the correct way to $\phi$ ), and in terms of the possession of relevant abilities. Take the case of knowing how to play the first movement of Beethoven's Moonlight Sonata. If it has recently been announced by reputable sources (the BBC, say) that it has been discovered that this movement has been misplayed for centuries, but I know that you have yet to hear this information, then I will not regard you as an epistemic peer with regard to knowing how to play this sonata. You thus do not count as an epistemic peer on evidential grounds. In contrast, if I am aware that, unlike myself, you are completely unable to play the piano, then you will not count as an epistemic peer with regard to knowing how to play this sonata because you lack relevant expertise in this respect. You thus do not count as an epistemic peer on ability grounds. ${ }^{14}$

With these points in mind, let us return now to the broad insight articulated above- that the intellectually virtuous agent should thus exhibit at least some degree of epistemic humility in the face of a disagreement with a recognized epistemic peer. What will this involve in a know- 
how disagreement? As it turns out, this question turns importantly on the prior issue-hotly debated in recent mainstream epistemology—of what it is in virtue of when one knows how to do something. ${ }^{15}$

According to one prominent proposal in the literature on knowledge-how -known as intellectualism - knowledge-how just is a kind of knowledge-that, and so knowing how to do something is just a matter of knowing some relevant proposition. ${ }^{16}$ In particular, the intellectualist's view is, in short, that $S$ knows how to $\phi$ when for some way $w, S$ knows that $w$ is the way for $S$ to $\phi$. For example, the intellectualist submits that when David knows how to play the first movement of Beethoven's Moonlight Sonata, it will be in virtue of David knowing that some way $w$ is a way for him to play the piece. ${ }^{17}$ There are further elements that some intellectualists add to this proposal—for instance, Jason Stanley (2011) notably insists that the relevant item of propositional knowledge must be known under what he calls a 'practical mode of presentation. ${ }^{, 18}$ But we'll set these details aside and concentrate on the core element of the thesis - that one knows how to do something just in case one stands in a propositional relation to some (suitably described) fact.

If the intellectualist about knowledge-how is right—such that knowledge-how just is propositional knowledge - then it follows that know-how disagreements between recognised epistemic peers will be just a special case of the more general class of propositional disagreements between recognised epistemic peers. Hence the requirements of epistemic humility, in the face of a know-how disagreement, will be the requirements of epistemic humility in the face of any more general propositional disagreement. That is, it will simply amount to lowering one's confidence with regard to the target proposition, a proposition which in this case is about some way being the way for one to do something.

To make this point more concrete, let's suppose there are just two incompatible ways to play the first movement of the Moonlight Sonata (say, $w$ is the correct sequence of notes, as written by Beethoven, and $w^{*}$ is like $w$ but contains a 'bad' series of notes, with a few sequences switched around). If the intellectualist about knowledge-how is correct, then David, upon discovering that his 'piano-peer' (Pete) insists that $w^{*}$, rather than $w$, is the way to play the first movement of the Moonlight Sonata, should no longer remain just as confident as he was before regarding the proposition that $w$ is the way to play the piece. ${ }^{19}$ And this will just amount to David lowering his credence (to at least some degree) that $w$ is true.

In contrast, what is involved in manifesting epistemic humility in know-how disagreements will be less straightforward for those proposals that reject the intellectualist's reduction of knowledge-how to knowledge-that. Anti-intellectualists (e.g., Ryle 1945; 1949; Poston 
2009; Carter \& Pritchard 2013; 2014) deny the intellectualist's key claim that when one knows how to do something (e.g., to ride a bike), this is in virtue of one's propositional attitudes (e.g., one's propositional attitude with regard to some proposition about a way to $\phi$ ). Call this the antiintellectualist's negative claim. In conjunction with this negative claim, anti-intellectualists also submit a positive claim — vir., that when one knows how to $\phi$ it will be in virtue of one's relevant abilities. $^{20}$

We'll engage with the positive claim of the proposal in more detail in the next section. But here it is important to note an interesting and thus-far overlooked implication of the antiintellectualist's negative claim, which is that it follows from this claim that know-how disagreements won't be just a matter of disagreeing about the truth of a proposition. After all, on the anti-intellectualist's proposal, neither side's putative knowledge-how will be in virtue of a propositional attitude which they hold. This turns out to be crucial from the perspective of determining how, according to anti-intellectualism, one should manifest intellectual humility in the face of a know-how disagreement with an epistemic peer. What it means, in short, is that to the extent that some kind of intellectual humility is plausibly required of the virtuous agent in the face of a recognised peer disagreement about how to $\phi$, the requisite humility cannot consist (as it will for the intellectualist) simply in belief revision. But then, in virtue of what will it consist, exactly?

\section{THE PROBLEM CLARIFIED}

We saw in the previous section how the anti-intellectualist's negative claim generated a difficult question. If the anti-intellectualist is right that it's not the case that when one knows how to do something this will be in virtue of one's propositional attitudes, then how, exactly, is intellectual humility to be manifested in the face of a know-how disagreement with a recognized epistemic peer?

Attending in closer detail to the anti-intellectualist's positive claim will serve to sharpen the puzzle further. Recall that the key thrust of the anti-intellectualist's positive claim is that when one knows how to do something, it will be in virtue of one's $\phi$-relevant abilities (as opposed to propositional attitudes). ${ }^{21}$ While we can make sense of revising credences, it's prima facie puzzling how one might go about, in an analogous manner, 'revising abilities'. Abilities, after all, aren’t simply objects of propositional attitudes nor are they subject to credence assignments-and so revision looks, on first blush, not easy to make sense of. This spells trouble for the anti- 
intellectualist insofar as she aims to preserve the plausible insight that in the face of disagreement with a recognised epistemic peer the epistemically virtuous agent should adopt some stance of intellectual humility.

We shall propose a solution to the problem in the next section, but first we want to locate the problem sketched for anti-intellectualist in a broader context. Until quite recently, intellectualist views tended to be defended on largely linguistic grounds, while (contemporary) anti-intellectualist views, in contrast, were often motivated by appeal to contemporary cognitive science. ${ }^{22}$ However, there has been some recent attempts in the literature to break through this impasse by appealing to strictly epistemological considerations to adjudicate the dispute (e.g., Poston 2009; Carter \& Pritchard 2013; 2014; Cath 2014). In particular, it has been suggested that knowledge-how has a range of epistemic properties which knowledge-that lacks, and that this is at odds with intellectualism. ${ }^{23}$ But if it turns out that intellectualism, but not anti-intellectualism, has the resources to make sense of how an epistemically virtuous agent should adopt a stance of intellectual humility in the face of a recognized peer disagreement about know-how, then this will obviously undermine the anti-intellectualist's claim that it has an advantage over intellectualism on specifically epistemological grounds.

\section{A SOLUTION}

Resolving this problem on behalf of anti-intellectualism will require us to fill in the blank in the following analogy:

Belief is to lowering credence as ability is to

Lowering credence is a way to adopt a stance of epistemic humility with regard to belief. But what is a way to adopt a stance of epistemic humility with regard to ability?

It will help to make the issue more concrete by considering an example of a know-how disagreement involving recognised epistemic peers. Suppose that there is one and only one way to dismantle the X2000 Bomb, a way that involves a sequence of cutting 20 wires in a particular order, where the $20^{\text {th }}$ step involves cutting a red wire (no other colour will do). Call going through this particular sequence of steps way ' $w$ '. Reddy and Whitey (who take each other to be roughly equals with respect to all skills and evidence which are relevant to bomb dismantling) discover that they disagree about how to dismantle the X2000. Furthermore, suppose that it is common ground between Reddy and Whitey that one and only one sequence will suffice (they do not think, for instance, that there is more than one equally valid sequence of wires that can be cut 
with the result that the X2000 will be dismantled). Reddy insists to Whitey that $w$ is the way to dismantle the bomb, with the cutting of the red wire as the $20^{\text {th }}$ step. Whitey insists, however, that Reddy's way wouldn't work, and maintains that cutting the white wire (in the final step) is the way to go.

With this case in mind, and to get a feel for what would constitute a plausible answer to the 'analogy' problem, let's consider first a 'bad' answer-bad in the sense of featuring an overconcessive response. Suppose that, upon finding that Whitey (his recognized epistemic peer) employs a different and incompatible way of dismantling the X2000 than Reddy himself is disposed to employ, Reddy entirely 'abjures' way $w$, in that he avows to never employ way $w$ again in the relevant context (e.g., when faced with the obstacle of dismantling an X2000). Instead, he resolves that in future situations he will simply employ Whitey's way, which involves cutting the white wire.

This response surely involves too much intellectual humility - to a fault. ${ }^{24}$ In fact, to the extent that intellectual humility is widely taken to be an intellectual virtue, we should expect intellectually virtuous humility to occupy something of a mean between, on the one hand, overconcessiveness and, on the other hand, over-rigidness, or dogmatism. ${ }^{25}$ As Robert Roberts and W. Jay Wood $(2007,188)$ have put it, being willing to very easily substantially overturn one's own intellectual perspective constitutes a vicious kind of 'intellectual flaccidity'. ${ }^{26}$

Just consider, after all, the structurally analogous move from the perspective of the intellectualist about knowledge-how. Intellectually virtuous humility will surely not require that when two recognised epistemic peers disagree with regard to a proposition specifying a way to do something, that each must believe outright the proposition one's disagreeing epistemic peer believes. And, by parity of reasoning, we should not think that in the situation described Reddy should simply employ Whitey's way in future circumstances.

Consider, by contrast, the over-rigid counterpart to the over-concessive move just considered. That is, should Reddy continue to employ his previous method (of cutting the red wire on the $20^{\text {th }}$ step) just as before, and to the very same extent, even after he has learned that Whitey, his recognised epistemic peer, employs a different and incompatible method? But this would involve no intellectual humility on his part, at least with regard to the issue of how to dismantle the X2000. As far as bomb dismantling goes, we might well call such a person intellectually arrogant, and dangerously so, given the evident seriousness of the endeavour in question.

Against these two extremes, we claim that the anti-intellectualist has the following much more reasonable 'middle way' available. This is that what is required of the epistemically virtuous 
agent when confronted with recognised epistemic peer disagreement regarding knowing how to $\varphi$ is plausibly that thereafter she should be disposed to employ her way of $\varphi$-ing across a narrower range of practical circumstances than beforehand. We'll get to the matter of 'how much' narrower shortly.

First, consider what it would be to satisfy this constraint. We will stipulate that prior to the disagreement with Whitey, Reddy would (very confidently) have been willing to employ way $w$ to dismantle an X2000 in not merely low-risk scenarios (e.g., when operating on a toy model bomb, in private), and medium-risk scenarios (e.g., where there's only a .0001 chance that the bomb is genuine, rather than a fake), but also in very high-risk, life-or-death situations. For example, to set Reddy's confidence especially high, suppose even further that Reddy would rely on $w$ in a high-risk case by volunteering to dismantle the bomb via way $w$ even when he is fully aware that there are other experts around. This circumstance is plausibly one in which it is reasonable to employ a method only if one is very perhaps maximally-confident that this method is effective.

Employing his way $(w)$ of dismantling the X2000 across a narrower range of circumstances, for Reddy, will thus involve being unwilling to rely to the same extent on his way of $\varphi$-ing in situations like the (very high-risk) situation just described. It will therefore at least involve not volunteering in such a circumstance and, plausibly, will involve quite a bit more caution (e.g., volunteering only if none of the other experts will). Importantly, however, exhibiting an appropriate degree of intellectual humility does not mean that Reddy must (after his disagreement with Whitey) never rely on $w$ in any circumstances-rather, what we are proposing is that the range of practical circumstances in which Reddy relies on $w$ should be comparatively narrower than before, and that the circumstances in which Reddy should be less inclined to no longer employ $w$ will correspond with the circumstances of highest risk in which he was previously prepared to employ $w$.

Thus, if we suppose that Reddy was originally extremely confident in $w$, then (as we indicated) he will no longer employ $w$ in life-or-death circumstances (at least where there are other experts around). This leaves open, though, that Reddy might satisfy the demands of intellectual humility while continuing to rely on $w$ in lower-risk scenarios, such as scenarios of medium-level risk. But if we were to instead stipulate that Reddy originally occupied a different starting point, confidence-wise, with respect to $w$ (suppose he originally would have relied on $w$ in scenarios of up to only medium-level risk, but not high-level risk), then intellectual humility requires that Reddy now be less inclined to rely on $w$ in situations of medium-level risk, while he can continue to rely on $w$ in scenarios of low-level risk. 
There are, at this point, three observations we that we would like to highlight. The first is that this proposal constitutes a way of exercising intellectual humility in the face of know-how disagreements that is available to anti-intellectualist accounts of knowledge-how. There is thus not a specific epistemological problem that faces anti-intellectualist accounts on this score, and hence intellectualist treatments of knowledge-how are not better placed to account for intellectual humility in the face of know-how disagreements.

The second observation is one of analogy. We have proposed that the demands of intellectual humility will be sensitive to the prior range of practical circumstances under which one is prepared to employ one's way of $\varphi$-ing. This is why, for instance, we've proposed that if one would originally have relied on one's way of $\varphi$-ing across an especially wide range of practical circumstances (including very high-risk circumstances), then one might then still be permitted to rely (after recognising the peer disagreement) on one's way of $\varphi$-ing in situations of medium-level (but not extremely-high) practical risk, even though this will not be the case for one who originally (prior to the recognised peer disagreement) would have relied on his way of $\varphi$-ing in only medium-range levels of practical risk.

The observation by analogy is that a parallel insight is entirely natural in the literature on epistemic peer disagreement as regards belief revision. Consider two individuals, A and B, both recognised epistemic peers and disagreeing about a proposition, $p$. While A had an initial credence in $p$ of $1, \mathrm{~B}$ had an initial credence in $p$ of .5. In granting that the requirements of intellectual humility mean that A and B should both lower their credences in $p$, we should also take into account their very different initial credences. In particular, A will presumably still retain a higher credence in $p$ than $\mathrm{B}$ even after the disagreement.

The third observation concerns the complicated matter of just how much narrower the practical circumstances should be in which an agent will continue to employ her way of $\varphi$-ing, subsequent to the epistemic peer disagreement. Obviously, for consistency's sake, any answer here should be broadly analogous to what is said in the doxastic case. We are not taking a stand in the doxastic case - this remains a live and on-going debate—and by the same token we are not proposing a definitive answer to this question in our discussion here. One option, for example, might be to follow Elga (2007) in going for a 'split the difference' view, which would result in a quite dramatic reduction in the practical circumstances in which an agent will continue to employ her way of $\varphi$-ing. But there are of course other options available, some of them less conciliatory.

We think that the important point to bear in mind when it comes to this issue is that the agent needs to call on her virtues — and on her practical wisdom in particular —in order to determine the extent of conciliation required by the disagreement in play. As with all exercise of 
the virtues, particularly in practical contexts, this is unlikely to be a straightforward matter and certainly won't submit to simple rules which an agent can follow. Virtue requires good judgement, and the right judgement is often very specific to the case in hand. This is one of the reasons why virtuous behaviour is so difficult to codify, if indeed it can be (in any useful way) codified at all. In any case, the issues raised here take us well beyond the remit of this paper, since they concern the very nature of the virtues and how to exercise them. ${ }^{27}$

\section{CONCLUDING REMARKS}

Our primary aim in this paper has been to show that running alongside the lively contemporary debate about propositional epistemic peer disagreement there is also a completely neglected parallel debate about know-how disagreements. Moreover, we've also shown that unless intellectualism about knowledge-how is correct, then there is a prima facie problem with accounting for such disagreements. In particular, it is on the face of it hard to see how antiintellectualist accounts of knowledge-how can accommodate the requirements of intellectual humility in the face of recognised epistemic peer disagreements regarding knowledge-how. This potentially constitutes a strike against anti-intellectualist accounts on epistemological grounds, which is surprising given that anti-intellectualist views have recently been specifically defended on epistemological grounds. We have shown, however, that there is a viable account of the requirements of intellectual humility in the face of know-how disagreements which is available to anti-intellectualist proposals. There is thus no reason to prefer intellectualist accounts of knowledge-how, at least on this score. ${ }^{28}$ 


\section{REFERENCES}

Baehr, J. (2011). The Inquiring Mind: On Intellectual V irtues and Virtue Epistemology, Oxford: Oxford University Press.

Bengson, J., \& Moffett, M. (2011a). 'Two Conceptions of Mind and Action: Knowing How and the Philosophical Theory of Intelligence', Knowing How: Essays on Knowledge, Mind, and Action, (eds.) J. Bengson \& M. Moffett, 3-58, Oxford: Oxford University Press.

- (2011b). 'Nonpropositional Intellectualism', Knowing How: Essays on Knowledge, Mind, and Action, (eds.) J. Bengson \& M. Moffett, 161-95, Oxford: Oxford University Press.

Brogaard, B. (2008). 'Knowledge-The and Propositional Attitude Ascriptions', Grazer Philosophische Studien 77, 147-90.

— (2009). 'What Mary Did Yesterday: Reflections on Knowledge-wh', Philosophy and Phenomenological Research 78, 439-67.

- (2011). 'Knowledge-How: A Unified Account', Knowing How: Essays on Knowledge, Mind, and Action, (eds.) J. Bengson \& M. Moffett, 136-60, Oxford University Press, Oxford.

Carter, J. A., \& Pritchard, D. H. (2013). 'Knowledge-How and Epistemic Luck', Noûs (Online First, DOI: 10.1111/nous.12054).

- (2014). 'Knowledge-How and Cognitive Achievement', Philosophy and Phenomenological Research (Online First, DOI: 10.1111/phpr.12094).

Carter, J. A. (Forthcoming). 'Group Peer Disagreement', Ratio.

Cath, Y. (2014). 'Revisionary Intellectualism and Gettier', Philosophical Studies (Online First, DOI: 10.1007/s11098-013-0263-y).

Christensen, D. (2007). 'Epistemology of Disagreement: The Good News', The Philosophical Review $116,187-217$.

— (2009a). 'Introduction: The Epistemology of Disagreement', Episteme 6, 231-32.

(2009b). 'Disagreement as Evidence: The Epistemology of Controversy', Philosophy Compass 4, 756-67.

Christensen, D., \& Lackey, J. (2013). The Epistemology of Disagreement: New Essays, Oxford: Oxford University Press.

Conee, E. (2009). 'Peerage', Episteme 6, 313-23.

Elga, A. (2007). 'Reflection and Disagreement', Nô̂s 41, 478-502.

Feldman, R. (2007). 'Reasonable Religious Disagreements', Philosophers Without Gods: Meditations on Atheism and the Secular, (ed.) L. Anthony, 194-214. Oxford: Oxford University Press.

Feldman, R., \& Warfield, T. (eds.) (2009). Disagreement, Oxford: Oxford University Press.

Glick, E. (2011). 'Two Methodologies for Evaluating Intellectualism', Philosophy and Phenomenological Research 83, 398-434.

— (2013). 'Practical Modes of Presentation', Noûs, (Online First, DOI: 10.1111 /nous.12052).

Hales, S. (2014). 'Motivations for Relativism as a Solution to Disagreements', Philosophy 89, 63-82.

Jenkins, C. S. (2014). 'Merely Verbal Disputes', Erkenntnis 79, 11-30.

Karttunen, L. (1977). 'Syntax and Semantics of Questions', Linguistics and Philosophy 1, 3-44.

Kelly, T. (2005). 'The Epistemic Significance of Disagreement', Oxford Studies in Epistemology 1, 167-96.

Lackey, J. (2008). 'A Justificationist View of Disagreement's Epistemic Significance', Proceedings of the XXII World Congress of Philosophy 53, 145-54.

MacFarlane, J. (2007). 'Relativism and Disagreement', Philosophical Studies 132, 17-31.

McDowell, J. (1979). 'Virtue and Reason', The Monist 62, 331-50.

Poston, T. (2009). 'Know How to Be Gettiered?', Philosophy and Phenomenological Research 79, 74347.

Pritchard, D. H. (2012a). 'Anti-Luck Virtue Epistemology', Journal of Philosophy 109, 247-79. 
- (2012b). 'Disagreement, Scepticism, and Track-Record Arguments', Disagreement and Skepticism, (ed.) D. Machuca, ch. 8, London: Routledge.

Pritchard, D. H., Millar, A., \& Haddock, A. (2010). The Nature and Value of Knowledge: Three Investigations, Oxford: Oxford University Press.

Roberts, R. C., \&Wood, J. W. (2007). Intellectual Virtues: An Essay in Regulative Epistemology, Oxford: Oxford University Press.

Ryle, G. (1945). 'Knowing How and Knowing That', Proceedings of the Aristotelian Society 46, 1-16.

- (1949). The Concept of Mind, Chicago, IL: University of Chicago Press.

Stalnaker, R. (2002). 'Common Ground', Linguistics and Philosopby 25, 701-21.

Stanley, J. (2011). Know How, Oxford: Oxford University Press.

Stanley, J., \& Krakauer, J. W. (2013). 'Motor Skill Depends on Knowledge of Facts', Frontiers in Human Neuroscience 7, 503.

Stanley, J., \&Williamson, T. (2001). 'Knowing How', Journal of Philosophy 98, 411-44.

van Inwagen, P. (1996). 'It Is Wrong, Everywhere, Always, for Anyone, to Believe Anything Upon Insufficient Evidence', Faith, Freedom and Rationality, (eds.) J. Jordan \& D. HowardSnyder, 137-54, Savage, MD: Rowman \& Littlefield.

Wallis, C. (2008). 'Consciousness, Context, and Know-How', Synthese 160, 123-53. 


\section{NOTES}

1 For a discussion of various ways of resolving disagreements, see Hales (2014).

2 See Kelly (2005) and van Inwagen (1996) for some notable resistance to this view. See also Pritchard (2012b). Note, however, that neither Kelly nor van Inwagen reject the idea that some degree of conciliation is epistemically appropriate in disagreements with epistemic superiors, and so neither maintains the strong thesis that disagreements are never epistemically significant.

3 See Lackey (2008) for the view that epistemic peerhood should be assessed along both an evidential dimension and also a cognitive ability dimension. For a more general discussion of epistemic peerhood, see Conee (2009).

${ }^{4}$ See, for example, Elga $(2007,478)$ for expression of the point that disagreement with epistemic inferiors needn't be epistemically significant.

5 In what follows we will, for simplicity, take a disagreement between recognised epistemic peers as involving both parties recognizing the other as an epistemic peer. Strictly speaking, however, all that is relevant for our purposes is that the party in the disagreement who is our particular focus recognizes the other as an epistemic peer; she need not herself be recognised by the other party as an epistemic peer.

${ }^{6}$ Elga's 'splitting the difference' proposal is framed in terms of credences. Hence, if A's initial credence with regard to $p$ is 1 and B's credence is .5, then A and B should both adjust their credences to .75. Feldman (2007), by contrast, frames the issue in terms of the 'triad' of doxastic attitudes of belief, disbelief, and withholding. Feldman's view is that for an individual who believes $p$ and discovers an epistemic peer believes not- $p$, A should move from the attitude of belief to the attitude of withholding. Note that exhibiting epistemic humility does not require taking on board either of these positions. As we've framed it, one demonstrates at least some epistemic humility so long as one does not continue believing the target proposition to the same extent. There are, of course, a range of positions available that fall under this general idea.

7 See, for instance, Kelly $(2005, \$ 1)$.

8 For a representative sample of literature in this regard, see Christensen (2009a), Feldman \& Warfield (2009), and Christensen \& Lackey (2013).

9 See also Christensen $(2009 b, 757)$. As he puts it, such cases are ones "where one comes to believe $p$ on the basis of certain evidence, and learns that one's apparent epistemic peer has reached the opposite conclusion on the basis of the same evidence."

10 To dispel a potential confusion here: we have said that most disagreements are propositional disagreements, though we've also characterized such disagreements as disagreements about whether $p$. Following received thinking about the semantics of wh-expressions (e.g., Karttunen 1977), we are taking it that one knows whether $p$ iff one knows, for some proposition $\mathrm{x}$, that $\mathrm{x}$ is the answer to some contextually relevant wh-question. Thus, disagreements about whether $p$ are fundamentally propositional disagreements.

11 We are not here attempting to provide a full account of what it is for a disagreement to be genuine, as opposed to, say, 'merely verbal'. In this respect, we're following the norm in the literature to accept as genuine exchanges where prima facie mutually precluding positions are advanced. The simplest such case will be on the 'assert/deny' model, where $\mathrm{A}$ asserts that $p$ and $\mathrm{B}$ denies that $p$. For some critical discussion of this simple view, see Karttunen (1977). See also Jenkins (2014) for helpful discussion on the difference between bona fide disagreements and merely verbal disputes.

12 We are understanding 'common ground' here in the standard way, along the lines of Stalnaker (2002).

13 Note, however, that what we've said here is compatible with recognizing that know-how disagreements can be genuine even when, in fact, there are multiply valid ways to $\phi$. Suppose that (as is very plausible) there are equally valid ways to drive from New York to Los Angeles; there is no unique correct way to do this, simpliciter. (For example, some routes might be best, from the perspective of safety, while others are best with respect to making good time, and so on). Even in such a case, we can quite easily imagine two individuals arguing (without taking into account any of these factors) in such a way that each proposes her own method as the correct way to $\phi$, and that the other person's method is wrong. In this case, it is common ground between A and B that there is one unique correct way of $\phi$-ing, even though there is not, in fact, a unique correct way of $\phi$-ing.

14 Note that one difference between epistemic peerhood in the case of know-how disagreement, in contrast to propositional disagreements, is that in the latter case the abilities at issue are specifically cognitive, whereas they need not be in the former case (e.g., the issue could be whether one's interlocutor has piano-playing abilities, as in the example just cited). To this extent, epistemic peerhood when it comes to know-how disagreement is a little less epistemic than the corresponding notion at issue in propositional disagreements.

15 The epistemology of knowledge-how has been revitalized in recent years, largely due to an influential paper by Stanley \& Williamson (2001) which argued against the received view_owing primarily to Ryle (1945; 1949)—that knowledge how is fundamentally different from knowledge-that. See Bengson \& Moffett (2011b) for a nice summary of the current state of play regarding knowledge-how.

16 At least, this 'reductive' claim is held by the most popular version of intellectualism in the recent literaturereductive intellectualism — according to which it's claimed both that: (i) when one knows how to $\phi$, this will be in virtue of one's propositional attitudes (e.g., in virtue of one's knowing some proposition); and (ii) that the metaphysical 
nature of knowledge-how is that of a relation between an agent and a proposition (such that the metaphysical nature of knowledge-how is that of a propositional knowledge relation). As Bengson \& Moffett (2011b) have noted, one counts as an intellectualist provided one accepts (i). Thus intellectualists, as such, needn't also embrace the further 'reductive' claim characterized by (ii). Bengson $\&$ Moffett take this line in embracing (i) and denying (ii). They think that although one knows how to $\phi$ in virtue of one's propositional attitudes, it's false that the metaphysical nature of knowledge-how is that of a relation between an agent and a proposition. Instead, on their intellectualist proposal the metaphysical relation is an objectual attitude relation between an agent and a way of $\phi$-ing. As such, Bengson $\&$ Moffett's proposal constitutes a 'non-reductive' variety of intellectualism. For the sake of our discussion we will be focusing on standard reductive versions of the view, which embrace both (i) and (ii).

17 We are taking it that one plays the first movement of the Moonlight Sonata only if one plays a particular sequence of notes. And so while there is some flexibility about how one can play the Moonlight Sonata (e.g., standing up as opposed to sitting down), there is no flexibility with regard to the series of notes played. If one plays a different series of notes, then while one might have played a variation on the Moonlight Sonata, one has not played the Moonlight Sonata.

18 For the original statement of this idea, see Stanley \& Williamson (2001, 429). See Glick (2013) for helpful discussion of this point.

19 We want to cut off at the pass a potential objection. Recall that according to a Stanley-style intellectualism, David knows how to $\phi$ only if he knows that some way $w$ is a way for David to $\phi$. Hence, it might be suggested that Pete is not disagreeing with David when he submits that $w$ is not the way for Pete to $\phi$. As such, know-how disagreements (by encoding the subject into the target proposition) are not genuine disagreements any more than are, say, contextualiststyle disagreements about predicates of personal taste, where the attributor's standard is encoded into the proposition expressed in the context of use. (See MacFarlane (2007) for further discussion of this kind of contextualism). We think this point can easily be set aside. This is because the kind of know-how disagreements that we are envisaging are precisely the kind of cases where the way to $\phi$ is genuinely in dispute. After all, David doesn't just hold that $w$ is the way for David to play the Moonlight sonata, but that it is also the way for Pete to play it too. 20 See Glick (2011) for discussion of anti-intellectualism as involving both these negative and positive claims.

21 See Ryle (1945) for one of the most familiar expressions of this idea. Part of Ryle's rationale in this paper, as well as in Ryle (1949, ch. 2), is that intellectualist accounts of intelligent action succumb to vicious regresses. While Ryle (1945) offers one such regress, his most famous regress argument is proposed in Ryle (1949). See Stanley (2011, ch. 1) for a sustained criticism of Ryle's regress arguments. Importantly, however, the plausibility of anti-intellectualism does not binge upon the success of Ryle's original regress arguments against the view.

22 See Stanley \& Williamson (2001) and Stanley (2011) for prominent examples of intellectualism being motivated on linguistic grounds. In terms of the relationship between anti-intellectualism and cognitive science, there is a growing precedent within cognitive science to advert to the distinction between declarative and procedural knowledge to forge a wedge between knowledge-that and knowledge-how, respectively. See, for instance, Wallis (2008). See also Stanley \& Krakauer (2013).

23 Poston (2009), for instance, has argued that knowledge-how and knowledge-that come apart because the former, but not the latter is resilient to being undermined by Gettier-style intervening luck. To the extent that this is right, it would mean that knowledge-how and knowledge-that have different epistemic properties, a claim that Stanley (2011, 216) himself acknowledges would count against the (reductive) intellectualist's proposal. Drawing on earlier work on knowledge and epistemic luck by Pritchard—see especially Pritchard, Millar \& Haddock (2010, chs. 1-4) and Pritchard (2012a) — Carter \& Pritchard (2013) make a rather different luck-based argument against intellectualism. They claim that while the kind of 'intervening' epistemic luck found in standard Gettier-style cases is (contra Poston) no more compatible with knowledge-how than it is with knowledge-that, the very different 'environmental' kind of epistemic luck is compatible with knowledge-how (but not also with knowledge-that). In addition, and drawing on the same body of previous work by Pritchard, Carter \& Pritchard (2014) further argues that knowledge-how and knowledge-that differ along dimensions of cognitive achievement. The latter, they argue, does not require cognitive achievement, while the former does.

24 We might say that what features here is something akin to the intellectual vice of epistemic under-confidence.

25 For some helpful recent work on intellectual humility as a virtue, see Baehr (2011)

26 Consider Roberts \& Woods' (2007) discussion of the following case, involving a student taking her first introductory course in philosophy:

"[...] she treats the survey as a smorgasbord at which she partakes with an appetite. Within a course of sixteen weeks she may have been a Platonist, an empiricist, a skeptic, a Cartesian, a Kantian, a utilitarian, a social contractor, a mind-body-dualist, a Berkeleyan idealist, a reductive materialist [...] she commits quickly to each theory, easily relinquishing its contrary, then passing on to the next. She is bright, but under the pressure of successive presentations of ideas, her intellectual character is too soft to hold onto a position." (Roberts \& Wood 2007, 188)

27 For an influential account of the exercise of virtue as essentially uncodifiable, see McDowell (1979). Although McDowell's focus here is on the ethical virtues, his proposal has application to the virtues more generally. 
28 Thanks to Emma C. Gordon, Michael Mi, Orestis Palermos, Ted Poston, Michael Slote, and Ernest Sosa. 